\title{
Amortecimento de Ondas Gravitacionais por Campos de Vegetação Flexível Parte I: Formulação do Modelo Teórico
}

\author{
Sávio Freire Lima; Claudio Freitas Neves \\ Programa de Engenharia Oceânica/COPPE/UFRJ - Rio de Janeiro, RJ \\ savio@lycos.com,neves@peno.coppe.ufrj.br \\ Nara Maria Luzzi Rosauro \\ Instituto de Pesquisas Hidráulicas/UFRGS - Porto Alegre, RS \\ nara.rosauro@ufrgs.br
}

Recebido: 14/11/06 - revisado: 20/04/07 - aceito: 11/05/07

\begin{abstract}
RESUMO
Este trabalho propõe uma nova abordagem para a determinação do amortecimento de ondas de gravidade propagando-se sobre campos de vegetação, que evita as simplificações implicitas nos modelos existentes e estende sua aplicação a espécies de grande flexibilidade, tais como Brachiaria subquadripora, encontradas freqüentemente nas margens de lagos artificiais no Brasil. Conceitualmente, o fenômeno foi dividido em dois processos distintos. Inicialmente, a resistência individual de uma haste é caracterizada através de um modelo mecânico que utiliza a velocidade relativa fluido-haste para calcular as forças exercidas pelas ondas sobre uma haste em função de um único parâmetro, o coeficiente de arraste. A seguir, estima-se a força média exercida pela coluna d'água sobre uma haste durante um período de onda e utilizam-se as equações do movimento integradas na vertical e promediadas no periodo da onda para avaliar o amortecimento gerado pelo conjunto. A reação do conjunto de hastes é função da força sobre uma haste, da densidade espacial de espécies vegetais e de um coeficiente de interação entre as hastes. A segunda parte deste trabalho apresentará os procedimentos experimentais para calibração do modelo e os respectivos resultados.
\end{abstract}

Palavras-chave: ondas gravitacionais; coeficiente de arraste.

\section{INTRODUÇÃOO}

A importância da vegetação aquática enquanto componente físico e biológico de ambientes costeiros e lacustres é amplamente reconhecida. Sua presença fornece alimento e abrigo para diversos organismos, modificando parâmetros ambientais e dissipando energia e turbulência de ondas e correntes (Bruno \& Kennedy, 2000; Mendez \& Losada, 2004). O grau de influência de uma espécie sobre a hidrodinâmica local será função do tamanho, densidade e distribuição espacial de seus indivíduos, que por outro lado são parâmetros controlados pela turbidez e pelos esforços mecânicos induzidos pelo escoamento (Madsen et al., 2001). Conseqüentemente, um estudo aprofundado do assunto deve contemplar a interatividade desta relação, onde a presença da vegetação influencia o escoamento, ao mesmo tempo em que este controla o seu desenvolvimento.
A natureza dos processos envolvidos no amortecimento de ondas por vegetação ainda não é totalmente compreendida e a quantificação dos esforços envolvidos está longe de ser satisfatória (Mork, 1996). Se, por um lado, não existe uma descrição teórica geral para estimar as modificações que elementos vegetais introduzem na propagação de ondas de gravidade, muitos são os estudos de caso que procuram representar o fenômeno para condições particulares. Alguns dos exemplos mais relevantes são: uso de algas artificiais para a reconstrução de bancos de areia (Price et al., 1968); uso de espécies naturais na fixação de bancos submetidos a ondas geradas pelo tráfego de embarcações (Coops et al., 1996); estudo dos impactos do desmatamento da Laminaria hyperborea na costa da Noruega (Mork, 1996); análise dos efeitos de campos de Macrocystis pyrifera na propagação de ondas no litoral da Califórnia (Elwany et al., 1995; Seymour, 1996); determinação dos esforços e limites para comprometimento da estrutura de diferentes espécies de algas 
(Denny et al., 1997; Gaylord \& Denny, 1997; Denny, 1999; Denny \& Gaylord, 2002; Gaylord et al., 2003).

No Brasil, embora não haja registros precisos do amortecimento de ondas de gravidade devido à presença de campos de vegetação ao longo da costa, sabe-se que bancos de algas pardas de grandes dimensões do gênero Laminaria estão presentes no trecho que se estende desde o sul da Bahia até o norte de Cabo Frio (Joly \& Oliveira Fo ${ }^{\circ}$ 1967). Além das algas, angiospermas marinhas da espécie Halodule wrightii são comuns em diversos pontos do litoral (Oliveira et al., 1997), formando extensas pradarias subaquáticas em algumas áreas da costa nordestina.

Nos rios e lagos, a interação do escoamento com a vegetação é mais evidente. Isto se deve principalmente à grande variedade de espécies adaptadas para ambientes que vão desde brejos até áreas totalmente submersas. De uma forma geral, estas espécies compreendem tanto macroalgas quanto plantas vasculares e são usualmente denominadas macrófitas aquáticas, estando amplamente difundidas ao longo dos corpos d'água brasileiros (Irgang \& Gastal Jr., 1996). Duas espécies particulares denominadas Brachiaria subquadrípara e Brachiaria mutica são encontradas em grandes quantidades nas margens de lagos e reservatórios de múltiplos usos (Carbonari et al., 2003). Porém, não foi encontrado na literatura nenhum registro de modelos que representem o efeito destas espécies sobre a propagação de ondas.

Em maio de 2003, foi realizado um experimento no lago de Ilha Solteira, no âmbito do Programa PROCAD de Cooperação Acadêmica da CAPES - Projeto "Amigos de Boussinesq - Rede Cooperativa de Pesquisa sobre Hidrodinâmica Costeira de Águas Rasas", entre a COPPE/UFRJ, o IPH /UFRGS e a FEIS/UNESP, com o objetivo de medir o espectro direcional das ondas em um ponto no meio do campo de vegetação e em outro ponto exterior, em local de profundidade igual a $5 \mathrm{~m}$. Filmagens subaquáticas e a colocação de uma mira topográfica debaixo d'água permitiram quantificar a magnitude dos deslocamentos das plantas, bem como qualificar o efeito das ondas sobre as plantas. Esta investigação era particularmente relevante para o lago de Ilha Solteira, onde estimativas preliminares indicavam ondas de até $1,25 \mathrm{~m}$ de altura e período de $6 \mathrm{~s}$ em direções onde a pista chegava a $40 \mathrm{~km}$ (Lima et al., 2003). Visitas ao local de fato comprovaram a erosão de margens, especialmente onde a vegetação era removida. Da mesma forma, as ondas induzidas pelo tráfego de embarcações também afetam o equilíbrio morfológico das margens. Tendo em vista que tal problema não se restringe ao lago de Ilha Solteira, a investigação sobre atenuação de ondas por meio de vegetação ou de elementos flexíveis artificiais desperta interesse por parte de empresas geradoras de energia elétrica e dos órgãos responsáveis pela manutenção de hidrovias.

\section{MODELOS EXISTENTES}

Embora alguns autores tenham conseguido bons resultados com modelos que simulam a área vegetada por uma camada de grande viscosidade (e.g. Price et al., 1968 e Mork, 1996), tradicionalmente as modelagens teóricas deste tipo de problema têm como base a determinação das forças induzidas pelas ondas nas plantas e suas reações. Camfield (1983) propôs que se representasse a vegetação como uma força de atrito de fundo resistente ao escoamento. Com o tempo, linhas de pesquisa voltadas para a abordagem das forças atuantes ao longo da estrutura da planta tiveram maior desenvolvimento (Dalrymple et al., 1984; Kobayashi et al., 1993; Mendez \& Losada, 2004). Contudo, a movimentação da planta é desprezada em todos estes trabalhos e na maioria dos estudos existentes.

Dalrymple et al. (1984) propuseram um modelo para representar as modificações impostas às ondas por áreas localizadas de perda de energia, tais como zonas de grande concentração de pilares ou vegetação submersa. As plantas foram representadas por elementos esbeltos que, por sua vez, introduziriam uma força de arraste sobre o escoamento na superfície de contato fluido-estrutura. Com base no balanço de energia ao longo da área vegetada, estes autores deduzem uma expressão para a taxa de decaimento da altura da onda, conhecida a sua altura $\mathrm{H}_{\mathrm{o}}$ na entrada do campo $(\mathrm{x}=0)$. A solução final para um fundo plano e horizontal é dada por uma expressão do tipo:

$\mathrm{H}(\mathrm{x})=\mathrm{H}_{\mathrm{o}}\left[\frac{1}{1+\beta \mathrm{x}}\right]$

onde:

$\beta=\frac{\mathrm{H}_{\mathrm{o}} \mathrm{N}_{\mathrm{h}} \mathrm{C}_{\mathrm{D}} \phi}{24 \mathrm{gc}_{\mathrm{g}} \mathrm{k} \pi} \frac{\left[\operatorname{senh}^{3}(\lambda \mathrm{kh})+3 \operatorname{senh}(\lambda \mathrm{kh})\right]}{3 \cosh ^{3}(\mathrm{kh})}\left(\frac{\mathrm{gk}}{\sigma}\right)^{3}$

é um coeficiente de amortecimento, $\mathrm{x}$ é a distância, $\mathrm{H}_{0}$ é a altura inicial da onda, $\mathrm{H}(\mathrm{x})$ é a altura da onda a uma distância $x, N_{h}$ é a densidade espacial da 
vegetação, $C_{D}$ é um coeficiente de arraste, $c_{g}$ é a celeridade de grupo da onda, $\mathrm{k}$ é o número de onda, $\sigma$ é a freqüência angular, h é a profundidade local, g é a aceleração da gravidade, $\phi$ é o diâmetro da haste e $\lambda$ é a razão entre a altura da vegetação e a lâmina d'água.

A expressão algébrica indicada na equação (1) é conseqüência do uso de uma potência quadrática no termo de velocidade da força de arraste, que acarreta o aparecimento de um termo cúbico da altura da onda na equação da taxa de dissipação de energia.

Kobayashi et al. (1993) criticaram esta abordagem, comum na maioria das pesquisas baseadas na equação da energia da onda, pois o escoamento local é estimado pela teoria linear e os efeitos produzidos pela presença da vegetação são representados somente por um termo de dissipação. Estes autores ressaltam que, embora muitos dos trabalhos desenvolvidos tenham encontrado resultados satisfatórios para os casos estudados, em situações onde a vegetação modifica o escoamento local significativamente, a hipótese da teoria linear e a aplicação de expressões obtidas sem vegetação são questionáveis. Estes autores propõem uma formulação obtida a partir das equações da continuidade e do movimento, visando uma solução mais realista para o problema de ondas monocromáticas de pequena amplitude propagando-se sobre áreas vegetadas. Eles supõem que a força de arraste $\overrightarrow{\mathrm{F}}_{\mathrm{D}}$ seja uma função linear da velocidade $\overrightarrow{\mathrm{u}}_{\mathrm{f}}$ do escoamento, cuja expressão é inserida como um termo de resistência horizontal na equação do movimento:

$\overrightarrow{\mathrm{F}}_{\mathrm{D}}=\rho_{\mathrm{f}} \mathrm{D} \overrightarrow{\mathrm{u}}_{\mathrm{f}}$

onde D é introduzido como um coeficiente de arraste linear a ser ajustado empiricamente e $\rho_{\mathrm{f}}$ é a massa específica do fluído.

Ao substituir esta expressão na equação do balanço de energia, chega-se a um termo quadrático em $\mathrm{H}$ referente à dissipação. Considerando novamente que $\mathrm{H}=\mathrm{H}_{0}$ para $\mathrm{x}=0$, a solução será satisfeita por uma expressão do tipo:

$\mathrm{H}(\mathrm{x})=\mathrm{H}_{\mathrm{o}} \mathrm{e}^{-\mathrm{k}_{\mathrm{i}} \mathrm{x}}$

Assim como $\beta, \mathrm{k}_{\mathrm{i}}$ é um coeficiente de amortecimento que deve ser determinado a partir de parâmetros tanto do escoamento quanto do campo de vegetação. A expressão obtida por Kobayashi et al. (1993) é:

$\mathrm{k}_{\mathrm{i}}=\frac{\mathrm{kD}}{2 \sigma} \frac{2 \lambda \mathrm{kh}+\operatorname{senh}(2 \lambda \mathrm{kh})}{2 \mathrm{kh}+\operatorname{senh}(2 \mathrm{kh})}$

onde as variáveis foram descritas anteriormente.

A adoção de um decaimento exponencial possui grande aceitação na literatura (e.g. Price et al., 1968; Mork, 1996), dada a conveniência de se trabalhar com um número de onda complexo. É interessante observar que a expansão em série do segundo membro da equação (1) e da função exponencial dada em (4) possibilita relacionar $\beta$ e $\mathrm{k}_{\mathrm{i}}$ :

$$
\begin{aligned}
& \frac{1}{1+\beta \mathrm{x}}=1-\beta \mathrm{x}+(\beta \mathrm{x})^{2}-(\beta \mathrm{x})^{3} \\
& \mathrm{e}^{-\mathrm{k}_{\mathrm{i}} \mathrm{x}} \cong 1-\mathrm{k}_{\mathrm{i}} \mathrm{x}+\frac{\left(\mathrm{k}_{\mathrm{i}} \mathrm{x}\right)^{2}}{2}-\frac{\left(\mathrm{k}_{\mathrm{i}} \mathrm{x}\right)^{3}}{3}
\end{aligned}
$$

Logo, para pequenos valores dos argumentos $\mathrm{k}_{\mathrm{i}} \mathrm{x}$ e $\beta \mathrm{x}$ (por exemplo, inferiores a 0,1 ), as expressões aproximam-se assintoticamente, e $\beta \cong \mathrm{k}_{\mathrm{i}}$. Recentemente, Mendez \& Losada (2004) estenderam a solução de Dalrymple et al. (1984) para a condição de águas rasas considerando um fundo com declividade constante $(\mathrm{m})$, obtendo:

$\mathrm{H}(\mathrm{x})=\mathrm{H}_{\mathrm{o}} \mathrm{K}_{\mathrm{s}} \mathrm{K}_{\mathrm{v}}$

onde:

$\mathrm{K}_{\mathrm{s}}=\left(\frac{\mathrm{h}_{\mathrm{o}}}{\mathrm{h}}\right)^{\frac{1}{4}}$ e $\mathrm{K}_{\mathrm{v}}=\frac{1}{1+\frac{2 \mathrm{~B}_{1}}{\mathrm{~m}} \mathrm{H}_{\mathrm{o}}\left(\mathrm{K}_{\mathrm{s}}-1\right)}$

onde:

$\mathrm{B}_{1}=2 \frac{\mathrm{C}_{\mathrm{D}}}{3 \pi} \phi \lambda \mathrm{N}_{\mathrm{h}}$

$\mathrm{C}_{\mathrm{D}}$ é o coeficiente de arraste $\mathrm{e} \mathrm{h}_{\mathrm{o}}$ é a profundidade do escoamento no início do campo de hastes.

Ao contrário das soluções anteriores, este caso apresenta a interferência de dois fenômenos: o aumento da altura da onda decorrente da redução de profundidade e o seu amortecimento pela presença do campo de vegetação. 
Todos os modelos apresentados até este ponto foram verificados através de experimentos e forneceram bons resultados para as características ensaiadas por seus autores, porém, em todos eles, o movimento da haste é negligenciado. Tal simplificação restringe a aplicação destes desenvolvimentos na determinação do amortecimento de ondas na presença de estruturas de grande flexibilidade. Embora Mendez \& Losada (1999) incluam o movimento das plantas no cálculo dos esforços hidrodinâmicos, as hastes são modeladas como vigas engastadas no fundo submetidas a um carregamento transversal, onde a linha elástica é aproximada pela hipótese de pequenos deslocamentos em função das propriedades biomecânicas da planta. Conseqüentemente, a solução do problema fica restrita a casos em que os movimentos da extremidade livre da haste sejam pequenos em relação ao seu comprimento.

De acordo com esta linha de abordagem, Vasco (2005) desenvolveu um modelo numérico para elementos rígidos ou semi-rígidos, engastados no fundo, incorporando um módulo de viscoelasticidade para simular o comportamento da vegetação. O autor, em suas conclusões, reforça a necessidade de aplicação de uma teoria não linear, tanto para o carregamento induzido pelas ondas, quanto para o movimento das plantas, e indica a necessidade de investigações experimentais sobre as propriedades biomecânicas de cada organismo.

De fato, as simplificações teóricas estão muito distantes do observado no campo de B. subquadrípara no lago de Ilha Solteira, pois durante a passagem das ondas, as amplitudes de oscilação da extremidade livre podiam atingir deslocamentos superiores a $10 \%$ do comprimento da haste.

Seria portanto desejável uma abordagem alternativa para o problema, que contemplasse o deslocamento das hastes e, conseqüentemente, a distribuição de velocidade no cálculo dos esforços exercidos sobre o escoamento. O presente trabalho propõe um modelo de amortecimento voltado para estruturas de grande flexibilidade e inclui uma teoria não linear para o cálculo das propriedades hidrodinâmicas do escoamento. A Tabela 1 mostra um resumo das principais características dos estudos apresentados, bem como do modelo proposto.

A maior parte dos trabalhos existentes propõe uma visão global do campo, onde se procura determinar somente o efeito final do conjunto de hastes sobre a onda. Mesmo que as expressões obtidas para o amortecimento tenham se baseado no cálculo das forças exercidas sobre um elemento isolado, a idéia de que a resistência total do campo seja dada pela soma das resistências individuais é questionável. Particularmente no caso de espécies flexíveis, onde os grandes deslocamentos favorecem o contato direto entre as hastes, novos esforços podem surgir modificando o comportamento idealizado.

Na prática, a eventual interação entre hastes é contemplada de forma implícita nos modelos de campo, pois a validação de expressões como as apresentadas em (1), (4) e (7) tradicionalmente é feita com base em curvas de amortecimento obtidas experimentalmente. Desta forma, o único parâmetro desconhecido na expressão do amortecimento, o coeficiente de arraste, é escolhido como sendo o valor que garanta o melhor ajuste entre as curvas experimental e teórica. Portanto, o valor obtido para este parâmetro estará englobando qualquer efeito adicional que influencie o amortecimento total, como a interferência entre hastes adjacentes.

Desta forma, a idéia da modelagem isolada de uma haste, que possibilite a determinação precisa dos coeficientes relacionados às forças atuantes localmente, torna possível a consideração explícita de um termo de interação entre hastes na equação final do amortecimento pelo campo. Esta perspectiva traz consigo uma abordagem inédita para este tipo de estudo. O próximo item ilustra a modelagem da resposta de uma haste.

\section{MODELO PROPOSTO}

A presente investigação procura modelar as modificações impostas à propagação de ondas de gravidade pela presença de conjuntos de elementos flexíveis que simulam o comportamento de algumas espécies de vegetação aquática. Entenda-se por elementos flexíveis, um arranjo de estruturas esbeltas, fixadas ao fundo, verticalizadas pelo empuxo e que oscilam em conseqüência dos esforços impostos pelo escoamento. Durante a propagação de ondas sobre campos formados por estes elementos, a troca de quantidade de movimento entre o fluido e a estrutura resulta na perda de energia do primeiro e, conseqüentemente, na redução da altura das ondas. Serão investigados os processos relacionados à hidrodinâmica da zona de decaimento, com o objetivo de se propor um modelo para estimar as modificações impostas a uma determinada onda em função de suas características iniciais e das propriedades geométricas das hastes.

Para tanto, o trabalho é dividido em duas etapas. Inicialmente, as equações do movimento são manipuladas de forma a expressar a variação da al- 
Tabela 1 - Principais modelos existentes para representar o amortecimento de ondas por campos de vegetação.

\begin{tabular}{|c|c|c|c|c|c|c|}
\hline Trabalho & $\begin{array}{l}\text { Influência das } \\
\text { plantas }\end{array}$ & $\begin{array}{l}\text { Movimento } \\
\text { das hastes }\end{array}$ & $\begin{array}{l}\text { Modelagem da } \\
\text { força }\end{array}$ & $\begin{array}{l}\text { Batimetria do } \\
\text { fundo }\end{array}$ & \begin{tabular}{ll|}
$\begin{array}{l}\text { Teoria } \\
\text { onda }\end{array}$ & de \\
\end{tabular} & $\begin{array}{l}\text { Interação } \\
\text { entre hastes }\end{array}$ \\
\hline $\begin{array}{l}\text { DALRYMPLE } \\
\text { et al. }(1984)\end{array}$ & $\begin{array}{l}\text { Dissipação de } \\
\text { energia }\end{array}$ & Não & $\begin{array}{l}\text { Arraste } \\
\text { quadrático }\end{array}$ & $\begin{array}{l}\text { Plano e horizon- } \\
\text { tal }\end{array}$ & Linear & Não \\
\hline $\begin{array}{l}\text { KOBAYASHI et al. } \\
(1993)\end{array}$ & $\begin{array}{l}\text { Força } \\
\text { distribuída }\end{array}$ & Não & Arraste linear & $\begin{array}{l}\text { Plano e horizon- } \\
\text { tal }\end{array}$ & Linear & Não \\
\hline $\begin{array}{ll}\text { MENDEZ } & \& \\
\text { LOSADA (1999) } & \\
\end{array}$ & $\begin{array}{l}\text { Força } \\
\text { distribuída }\end{array}$ & Sim & $\begin{array}{l}\text { Arraste linear e } \\
\text { inércia }\end{array}$ & $\begin{array}{l}\text { Plano e horizon- } \\
\text { tal }\end{array}$ & Linear & Não \\
\hline $\begin{array}{ll}\text { MENDEZ } & \& \\
\text { LOSADA }(2004) & \end{array}$ & $\begin{array}{l}\text { Dissipação de } \\
\text { energia }\end{array}$ & Não & $\begin{array}{l}\text { Arraste } \\
\text { quadrático }\end{array}$ & $\begin{array}{l}\text { Plano com de- } \\
\text { clividade cons- } \\
\text { tante }\end{array}$ & $\begin{array}{l}\text { Linear em } \\
\text { águas rasas }\end{array}$ & Não \\
\hline $\begin{array}{l}\text { Modelo proposto } \\
\text { (LIMA, 2005) }\end{array}$ & Força média & Sim & $\begin{array}{l}\text { Arraste } \\
\text { quadrático } \\
\text { (inércia) }\end{array}$ & $\begin{array}{l}\text { Plano e horizon- } \\
\text { tal }\end{array}$ & $\begin{array}{l}\text { Não linear } \\
\text { (função de } \\
\text { corrente) }\end{array}$ & Sim \\
\hline
\end{tabular}

* Referente à teoria utilizada no cálculo das forças hidrodinâmicas

tura da onda em função de uma força média na coluna d'água. Esta abordagem resgata a concepção de Longuet-Higgins (1976) ao introduzir o conceito de tensão de radiação, permitindo que se trabalhe explicitamente em termos de força, o que é fisicamente justificável posto que esta grandeza não se dissipa e pode ser obtida de forma direta.

Em uma segunda etapa, um modelo mecânico é desenvolvido para caracterizar hidrodinamicamente as hastes estudadas. As informações de força obtidas pela calibração deste modelo são inseridas diretamente nas equações modificadas do movimento, fornecendo uma expressão para o amortecimento das ondas (Lima et al. 2005).

Adotam-se as seguintes hipóteses simplificadoras:

1. para intervalos de tempo muito menores que o período da onda, a resultante de todas as forças atuantes em um nó é considerada constante;

2. devido à pequena resistência do material da haste à flexão, despreza-se o momento ao longo de seu eixo e os nós são considerados articulados;

3. a profundidade é constante e igual a h;

4. o movimento da haste se dá no plano vertical $x-z$, adotando-se um sistema de coordenadas cartesianas $(\mathrm{x}, \mathrm{z})$ estacionário e fixo no fundo, de modo que a superfície da água fica em $\mathrm{z}=\mathrm{h}+\eta$ (Fig. 1).

\section{Modelo de campo}

As equações da quantidade de movimento promediadas ao longo de um período de onda e integradas na vertical são reescritas como (Phillips, 1977):

$$
\begin{aligned}
& \frac{\partial}{\partial t} M_{i}+\frac{\partial}{\partial x_{j}}\left(U_{i} M_{j}\right)= \\
& p_{a t m}-\rho_{f} g(h+\bar{\eta}) \frac{\partial \bar{\eta}}{\partial x_{i}}-\frac{\partial}{\partial x_{j}} S_{i j}+\frac{1}{T} \int_{0}^{T} F_{i} d \tilde{t}
\end{aligned}
$$

com

$$
\begin{aligned}
& S_{i j}=-\frac{\tilde{M}_{i} \tilde{M}_{j}}{\rho_{f}(h+\bar{\eta})}+\frac{1}{T} \int_{0}^{T} \int_{-h}^{\eta}\left(\rho_{f} \tilde{u}_{j} \tilde{u}_{i}+p \delta_{i j}\right) d z d \tilde{t} \\
& -\frac{1}{2} \rho_{f} g(h+\bar{\eta})^{2} \delta_{i j}
\end{aligned}
$$

onde os índices i, j referem-se às direções $\mathrm{x}, \mathrm{z}$; $\mathrm{M}_{\mathrm{i}}$ - transporte de massa total, resultante de uma corrente arbitrária $(\hat{\mathrm{M}})$ e do transporte de massa associado à onda $(\tilde{M})$, definido por $M_{i}=\hat{M}_{i}+\tilde{M}_{i}$ onde

$\hat{M}_{i}=\frac{1}{T} \int_{0}^{T}\left(\int_{-h}^{\eta} \rho_{f} \hat{U}_{i} d z\right) d \tilde{t}$ e $\tilde{M}_{i}=\frac{1}{T} \int_{0}^{T}\left(\int_{-h}^{\eta} \rho_{f} \tilde{u}_{i} d z\right) d \tilde{t} ;$ 
$\mathrm{U}_{\mathrm{i}}$ - velocidade associada ao transporte de massa total devido à corrente e à onda, ou seja,

$U_{i}=\frac{M_{i}}{\rho_{f}(h+\bar{\eta})}=\frac{\hat{M}_{i}}{\rho_{f}(h+\bar{\eta})}+\frac{\tilde{M}_{i}}{\rho_{f}(h+\bar{\eta})} ;$

$\hat{\mathrm{U}}_{\mathrm{i}}$ - componente de velocidade associado a uma corrente permanente na escala da onda;

$\tilde{\mathrm{u}}_{\mathrm{i}}$ - componente de velocidade associado ao escoamento oscilatório;

$\tilde{\mathrm{t}}$ - escala de tempo da onda;

$\mathrm{t}$ - escala de tempo do escoamento promediado.

$\bar{\eta}$ - posição média da superfície livre dada por $\bar{\eta}=\frac{1}{\mathrm{~T}} \int_{0}^{\mathrm{T}} \eta \mathrm{d} \tilde{\mathrm{t}}$

$\mathrm{p}_{\mathrm{atm}}$ - pressão atmosférica;

$F_{i}$ - termo de forças distribuídas ao longo da coluna d'água.

O termo (10) depende somente das propriedades da onda. Suas componentes $\left(S_{i j}\right)$ formam um tensor conhecido como "tensor de radiação", que representa as forças induzidas pela não linearidade das ondas sobre o escoamento médio.

Considerando o escoamento médio como permanente, com ondas propagando-se paralelamente ao eixo $\mathrm{x}$, a retenção dos termos até $\mathrm{O}(\mathrm{H})^{2}$ reduz a equação (9) a:

$$
\begin{aligned}
& H \frac{\partial}{\partial x} H= \\
& -\frac{8}{\rho_{f} g(4 n-1)}\left[\frac{1}{4} \rho g H^{2} \frac{\partial}{\partial x} n+\rho_{f} g(h+\bar{\eta}) \frac{\partial}{\partial x} \bar{\eta}+\bar{F}_{x}\right]
\end{aligned}
$$

onde $\mathrm{H}$ é a altura da onda, $\mathrm{n}$ é a razão entre celeridade de grupo e a celeridade da onda e $\overline{\mathrm{F}}_{\mathrm{x}}$ foi introduzido para representar forças médias no período da onda que estejam sendo exercidas sobre a coluna d'água, como por exemplo a reação exercida pelas hastes.

\section{Termo de resistência do campo}

No caso específico de um campo onde os elementos são idênticos entre si, pode-se dizer que a força total exercida sobre o fluido por unidade de área horizontal será função da força exercida por uma única haste e da quantidade de hastes por metro quadrado. Escreve-se:
$\overline{\mathrm{F}}_{\mathrm{x}}=\alpha \mathrm{N}_{\mathrm{h}} \overline{\mathrm{F}}_{\mathrm{x}}^{1}$

sendo $\alpha$ um coeficiente de interação entre hastes, $\mathrm{N}_{\mathrm{h}}$ o número de hastes por unidade de área e $\overline{\mathrm{F}}_{\mathrm{x}}^{1} \mathrm{a}$ força média exercida na coluna d'água por uma única haste durante a passagem de uma onda. Devese observar que, ao contrário dos modelos usuais, não se está impondo que a resistência do campo seja igual à soma dos efeitos individuais das hastes. A introdução de $\alpha$ permite isolar efeitos decorrentes da ação conjunta das hastes do cálculo das forças individuais, cujo procedimento para sua determinação é apresentado a seguir.

\section{Resistência de uma haste}

Ao considerar uma haste flexível induzida ao movimento por um escoamento oscilatório onde o empuxo é o único agente restaurador, a força instantânea que atua ao longo da haste pode ser interpretada como a força de resistência que o elemento exerce sobre o escoamento, ou seja:

$\mathrm{F}_{\mathrm{x}}^{1}(\mathrm{t})=\int_{0}^{\mathrm{s}} \mathrm{q}_{\mathrm{Tx}}(\mathrm{x}, \mathrm{z}, \mathrm{t}) \mathrm{ds}$

onde $\mathrm{q}_{\mathrm{Tx}}$ é a força horizontal por unidade de comprimento atuando sobre o elemento em conseqüência do movimento relativo entre fluido e haste.

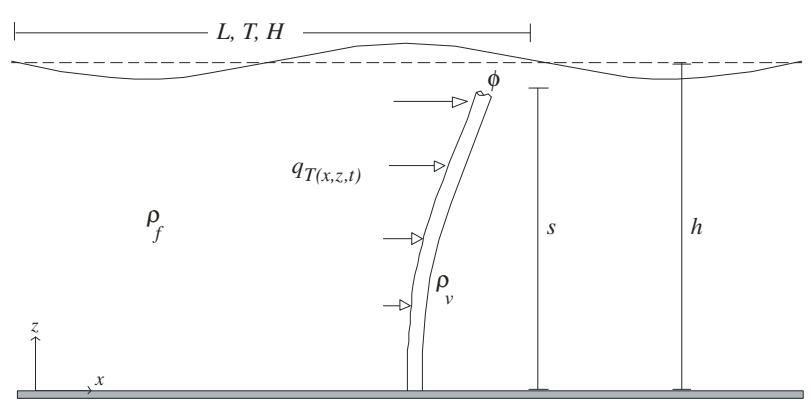

Figura 1 - Grandezas físicas envolvidas na indução do movimento da haste.

A priori, $\mathrm{q}_{\mathrm{T}}$ é dividida em duas componentes perpendiculares $\mathrm{q}_{\mathrm{Tx}}$ e $\mathrm{q}_{\mathrm{Tz}}$, porém, para efeito de balanço de forças sobre o escoamento médio integrado na vertical, somente a resistência horizontal é considerada. Esta abordagem é justificada para ondas em águas de intermediárias para rasas, em que a velocidade vertical é menor que a horizontal por um 
fator da ordem da esbeltez da onda. Por outro lado, pode ser uma simplificação exagerada não só para estudos em águas profundas, mas também para casos onde a posição média da haste esteja afastada do eixo vertical.

Deseja-se obter uma expressão para a força média exercida pelo fluido ao longo de toda a haste durante a passagem de uma onda. Esta força equivale exatamente à média temporal de $\mathrm{F}_{\mathrm{x}}^{1}$, ou seja:

$\overline{\mathrm{F}}_{\mathrm{x}}^{1}=\frac{1}{\mathrm{~T}} \int_{0}^{\mathrm{T}} \mathrm{F}_{\mathrm{x}}^{1}(\mathrm{t}) \mathrm{dt}$

A Figura 2 ilustra o comportamento medido de $\mathrm{F}_{\mathrm{x}}^{1}$ durante a passagem de uma onda, onde pode-se observar a assimetria da curva em relação à amplitude da força. Este desvio resulta em uma componente média no sentido de propagação da onda, e é exatamente o valor a ser inserido na equação (12).

Fica evidente que a determinação de $\overline{\mathrm{F}}_{\mathrm{x}}^{1}$ dependerá do conhecimento da evolução temporal das forças atuantes sobre todo o elemento flexível. Por outro lado, esta grandeza será conseqüência da velocidade relativa entre a haste e o fluido, que, a priori, não é conhecida, pois a velocidade da haste já é resultado do histórico dos esforços sobre ela aplicados. Neste ponto, propõe-se uma idealização do elemento flexível com o objetivo de desenvolver um modelo mecânico representativo de seu comportamento hidrodinâmico durante a passagem de ondas. Uma vez calibrado, o modelo fornece o histórico das forças exercidas ao longo da haste e conseqüentemente a equação (14) poderá ser resolvida.

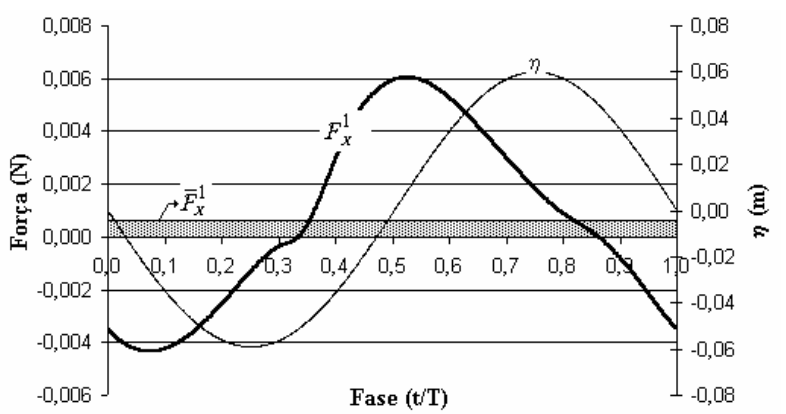

Figura 2 - Comportamento da força resultante sobre a haste ao longo de um período de onda. $O$ limite superior da área hachurada indica o seu valor médio no tempo.

\section{Concepção do modelo mecânico}

Considerando uma distribuição uniforme de massa ao longo do elemento, propõe-se discretizar a haste em segmentos, onde a força que atua em um pequeno trecho de comprimento $1_{n}$ é representada em um único nó. Diante da ação das ondas, em um dado instante t', cada elemento pontual terá uma velocidade $\vec{u}_{v\left(n, t^{\prime}\right)}$ e aceleração $\vec{a}_{v\left(n, t^{\prime}\right)}$. Neste mesmo instante, o fluido possui uma velocidade $\overrightarrow{\mathrm{u}}_{\mathrm{f}\left(\mathrm{n}, \mathrm{t}^{\prime}\right)}$ e aceleração $\overrightarrow{\mathrm{a}}_{\mathrm{f}\left(\mathrm{n}, \mathrm{t}^{\prime}\right)}$. A velocidade resultante entre cada nó e o escoamento em sua volta dará origem a forças hidrodinâmicas. A consideração fundamental do modelo é de que, para intervalos de tempo muito menores que o período da onda $(\Delta \mathrm{t}<<\mathrm{T})$, a resultante de todas as forças atuantes em um nó pode ser considerada constante. Desta forma, cada um dos nós será uniformemente acelerado desde o instante t' até t' $+\Delta \mathrm{t}$. A Figura 3 apresenta as simplificações adotadas na concepção do modelo.

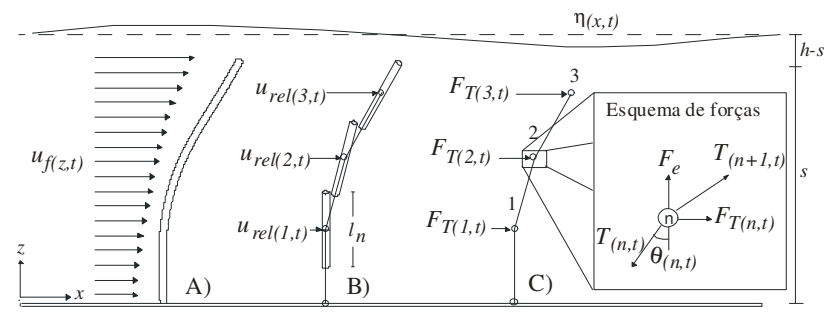

Figura 3 - Modelo mecânico para representar o comportamento de uma haste. A)Forma original da estrutura. B)

Divisão em segmentos. C) Balanço de forças nos nós.

Outra consideração importante é de que, devido à pequena resistência do material da haste à flexão, o momento ao longo de seu eixo pode ser desprezado e os nós são considerados articulados. Adota-se uma profundidade constante e igual a $h$. O sistema de coordenadas cartesianas $(\mathrm{x}, \mathrm{z})$ é estacionário e fixo no fundo, de modo que a superfície da água ficará em $\mathrm{z}=\mathrm{h}+\eta$. Considera-se ainda que o movimento da haste se dê somente no plano $\mathrm{x}-\mathrm{z}$.

Tendo a velocidade da haste como condição inicial, o modelo deverá calcular a aceleração atuante sobre cada nó durante o passo de tempo $\Delta$ t, que induzirá modificação em seu campo de velocidades, resultando em uma nova posição e condições iniciais para o passo seguinte. Desta forma, o modelo deverá fornecer séries temporais de posição, velocidade e forças ao longo de toda a haste. 
Para um fenômeno em duas dimensões, pode-se escrever as equações para o balanço de forças horizontais e verticais para um certo elemento n:

$\mathrm{F}_{\mathrm{Tx}\left(\mathrm{n}, \mathrm{t}^{\mathrm{t}}\right)}-\mathrm{T}_{\left(\mathrm{n}, \mathrm{t}^{\mathrm{\prime}}\right)} \operatorname{sen} \theta_{\left(\mathrm{n}, \mathrm{t}^{\prime}\right)}+\mathrm{T}_{\left(\mathrm{n}+1, \mathrm{t}^{\mathrm{t}}\right)} \operatorname{sen} \theta_{\left(\mathrm{n}+1, \mathrm{t}^{\prime}\right)}=M \mathrm{a}_{\mathrm{vx}\left(\mathrm{n}, \mathrm{t}^{\mathrm{t}}\right)}$

$\mathrm{F}_{\mathrm{Tz}\left(\mathrm{n}, \mathrm{t}^{\prime}\right)}+\mathrm{F}_{\mathrm{e}}-\mathrm{T}_{\left(\mathrm{n}, \mathrm{t}^{\prime}\right)} \cos \theta_{\left(\mathrm{n}, \mathrm{t}^{\prime}\right)}+\mathrm{T}_{\left(\mathrm{n}+1, \mathrm{t}^{\prime}\right)} \cos \theta_{\left(\mathrm{n}+1, \mathrm{t}^{\prime}\right)}=$

$\mathrm{Ma}_{\mathrm{vz}}\left(\mathrm{n}, \mathrm{t}^{\prime}\right)$

onde $\mathrm{F}_{\mathrm{Tx}\left(\mathrm{n}, \mathrm{t}^{\prime}\right)}$ e $\mathrm{F}_{\mathrm{Tz}\left(\mathrm{n}, \mathrm{t}^{\prime}\right)}$ correspondem às componentes horizontal e vertical da força $\mathrm{F}_{\mathrm{T}\left(\mathrm{n}, \mathrm{t}^{\prime}\right)}=\mathrm{q}_{\mathrm{T}\left(\mathrm{n}, \mathrm{t}^{\prime}\right)} 1_{\mathrm{n}}$ atuante nos nós, $\mathrm{T}_{\left(\mathrm{n}, \mathrm{t}^{\prime}\right)}$ é a interação entre nós adjacentes, $\theta_{\left(\mathrm{n}, \mathrm{t}^{\prime}\right)}$ representa o ângulo de inclinação do nó n com a vertical no instante t', $M$ corresponde à massa do elemento cilíndrico $\mathrm{M}=\rho_{\mathrm{v}} \mathrm{V}_{\mathrm{el}}$, onde $\mathrm{V}_{\mathrm{el}}$ é o volume do elemento, e $\mathrm{F}_{\mathrm{e}}$ é a força de empuxo sobre o segmento. $\mathrm{O}$ fato de se considerar as acelerações constantes ao longo do intervalo de tempo $\Delta \mathrm{t}$ permite aproximar os deslocamentos dos nós por:

$$
\begin{aligned}
& \mathrm{a}_{\mathrm{vx}\left(\mathrm{n}, \mathrm{t}^{\prime}\right)}=\frac{2}{\Delta \mathrm{t}}\left[\frac{\Delta \mathrm{x}_{\left(\mathrm{n}, \mathrm{t}^{\prime}\right)}}{\Delta \mathrm{t}}-\mathrm{u}_{\mathrm{vx}\left(\mathrm{n}, \mathrm{t}^{\prime}\right)}\right] \\
& \mathrm{a}_{\mathrm{vz}\left(\mathrm{n}, \mathrm{t}^{\prime}\right)}=\frac{2}{\Delta \mathrm{t}}\left[\frac{\Delta \mathrm{z}_{\left(\mathrm{n}, \mathrm{t}^{\prime}\right)}}{\Delta \mathrm{t}}-\mathrm{u}_{\mathrm{vz}\left(\mathrm{n}, \mathrm{t}^{\prime}\right)}\right]
\end{aligned}
$$

Substituindo (16) em (15), chega-se a um sistema com duas equações e três incógnitas em cada nó, a saber: $\mathrm{T}_{\left(\mathrm{n}, \mathrm{t}^{\prime}\right)}, \Delta \mathrm{x}_{\left(\mathrm{n}, \mathrm{t}^{\prime}\right)}$ e $\Delta \mathrm{z}_{\left(\mathrm{n}, \mathrm{t}^{\prime}\right)}$. Para tornar o problema determinado, propõe-se uma terceira equação baseada em uma condição constitutiva da haste. Considerando que os esforços impostos são de magnitude muito inferior aos necessários para causar deformações elásticas na estrutura, postula-se que a distância entre dois nós adjacentes, $1_{n}$, permaneça constante. Propõe-se a expressão:

$\left(\mathrm{x}_{\left(\mathrm{n}, \mathrm{t}^{\prime}\right)}-\mathrm{x}_{\left(\mathrm{n}-1, \mathrm{t}^{\prime}\right)}\right)^{2}+\left(\mathrm{z}_{\left(\mathrm{n}, \mathrm{t}^{\prime}\right)}-\mathrm{z}_{\left(\mathrm{n}-1, \mathrm{t}^{\prime}\right)}\right)^{2}=\mathrm{l}_{\mathrm{n}}{ }^{2}$

Deve-se reparar que todos os nós possuem conexão inferior, mas nem todos possuem uma conexão superior (caso do nó da extremidade superior), desta forma, a equação é escrita relacionando sempre o nó n com o nó n-1. Desenvolvendo e derivando em relação ao tempo, em seguida substituin- do os termos das derivadas por uma aproximação numérica e reorganizando os membros:

$$
\Delta \mathrm{x}_{\left(\mathrm{n}, \mathrm{t}^{\prime}\right)}=\left[\Delta \mathrm{x}_{\left(\mathrm{n}-1, \mathrm{t}^{\prime}\right)}+\frac{\Delta \mathrm{z}_{\left(\mathrm{n}-1, \mathrm{t}^{\prime}\right)}}{\operatorname{tg} \theta_{\left(\mathrm{n}, \mathrm{t}^{\prime}\right)}}\right]-\frac{\Delta \mathrm{z}_{\left(\mathrm{n}, \mathrm{t}^{\prime}\right)}}{\operatorname{tg} \theta_{\left(\mathrm{n}, \mathrm{t}^{\prime}\right)}}
$$

A Equação (18) relaciona $\Delta \mathrm{x}_{\left(\mathrm{n}, \mathrm{t}^{\mathrm{t}}\right)}$ a $\Delta \mathrm{z}_{\left(\mathrm{n}, \mathrm{t}^{\prime}\right)}$. Desta forma, para cada nó, tem-se três equações e três incógnitas, e para um sistema com $\mathrm{N}$ nós, ter-seá um total de $3 \mathrm{~N}$ equações e $3 \mathrm{~N}$ variáveis. A solução do sistema fornecerá as forças longitudinais de conexão entre os nós e os seus deslocamentos em um passo de tempo.

A força exercida pelo escoamento sobre uma estrutura vertical cilíndrica, é representada por uma equação do tipo:

$\overrightarrow{\mathrm{F}}_{\mathrm{T}}=\frac{1}{2} \underbrace{\mathrm{C}_{\mathrm{D}} \rho_{\mathrm{f}} \overrightarrow{\mathrm{A}}_{\mathrm{eq}} \overrightarrow{\mathrm{u}}_{\mathrm{rel}}\left|\overrightarrow{\mathrm{u}}_{\mathrm{rel}}\right|}_{(1)}+\underbrace{\mathrm{k}_{\mathrm{m}} \rho_{\mathrm{f}} \mathrm{V}_{\mathrm{el}} \overrightarrow{\mathrm{a}}_{\mathrm{rel}}}_{(2)}+\underbrace{\rho_{\mathrm{f}} \mathrm{V}_{\mathrm{el}} \overrightarrow{\mathrm{a}}_{\mathrm{f}}}_{(3)}$

onde $C_{D}$ é o coeficiente de arraste, $\overrightarrow{\mathrm{A}}_{\text {eq }}$ corresponde à área da face da estrutura normal ao vetor velocidade relativa entre o fluido e a haste, $\vec{u}_{\text {rel }}=\vec{u}_{f}-\vec{u}_{v}$, $\mathrm{k}_{\mathrm{m}}$ é o coeficiente de massa adicional e $\vec{a}_{\text {rel }}=\vec{a}_{f}-\vec{a}_{v}$ é a aceleração relativa entre o fluido e a haste no nó considerado.

A expressão (19) é uma generalização da Equação de Morison, para considerar o movimento da estrutura (Brebia \& Walker, 1979). O primeiro termo representa as forças de arraste; o segundo termo representa a parcela da força de inércia associada à parcela adicional de fluido que acompanha o movimento da haste, como se aparentemente fosse aumentado o volume do elemento; o terceiro termo é a parcela da força de inércia associada exclusivamente à aceleração do fluido. Embora a equação tenha origem empírica, tentativas de se obter formulações que melhor representem esforços hidrodinâmicos sobre estruturas não têm apontado para soluções mais realistas (Chakrabarti, 1987).

Durante o desenvolvimento do modelo, o movimento da haste foi filmado de cima do canal de ondas, revelando que oscilações transversais ao sentido de propagação das ondas não eram observadas. Por este motivo, a expressão adotada não contempla forças horizontais transversais ao escoamento.

$O$ coeficiente de arraste, $C_{D}$, será uma função do escoamento e das características da estrutura. $\mathrm{Na}$ literatura existem diversos trabalhos correlacio- 
nando o seu valor com parâmetros adimensionais, tais como o número de Reynolds e de KeuleganCarpenter. Já o coeficiente de massa adicional, $\mathrm{k}_{\mathrm{m}}$, depende da forma do objeto e muitos autores apresentam funções explicitas de seu valor para diferentes seções transversais. É comum, ainda, a aceleração da estrutura ser ignorada e as forças de inércia serem representadas por um único termo através da introdução de um novo coeficiente $\mathrm{C}_{\mathrm{M}}=1+\mathrm{k}_{\mathrm{m}}$. Buscando uma maior representatividade para os casos estudados neste trabalho, os coeficientes de força foram ajustados a partir de medições experimentais.

A obtenção da velocidade do escoamento nos nós para cálculo do modelo deve ser feita a partir de uma teoria de ondas que represente satisfatoriamente as características observadas nos locais de ocorrência da vegetação, que na maioria das vezes está próxima da margem. Esta característica aponta para ondas cujos termos não-lineares passam a ser importantes e, nestas condições, segundo Dean (1968), a Teoria da Função de Corrente é a mais indicada para representar o escoamento. Este autor constatou ainda que, em águas rasas, o cálculo da força de arraste a partir de teorias analíticas pode incorrer em erros exagerados.

Para a determinação das propriedades da onda durante as simulações com o modelo mecânico, optou-se pelo uso da Teoria da Função de Corrente, assim, a assimetria do campo de velocidades decorrente da não-linearidade das ondas passa a ser considerada no cálculo das forças hidrodinâmicas. A referida teoria consiste em uma aproximação em série, onde os coeficientes multiplicadores de cada termo são obtidos através de um método interativo de minimização do erro na condição de contorno dinâmica na superfície livre. Detalhes sobre a colocação do problema de minimização do erro para a obtenção dos coeficientes da série podem ser obtidos em Dean (1968) e Dean e Dalrymple (1984).

\section{Comentários finais}

O desenvolvimento apresentado nesta seção aliado a experimentos com o tipo de haste investigado permite uma análise criteriosa não só das forças exercidas sobre o escoamento por um indivíduo, como também da força total de resistência de um conjunto deles. Seria desejável a repetição do procedimento para diferentes condições de onda e características físicas da haste, de modo a produzir informações que possibilitassem o refinamento de um modelo conceitual específico para o amortecimento de ondas de gravidade em campos formados por estes elementos. Idealmente, os experimentos deveriam ser feitos com as próprias plantas, mas isto exigiria cuidados adicionais, não apenas para transportá-las de Ilha Solteira para o Rio de Janeiro, como também para adaptá-las a um novo ambiente com água clorada e diferentes condições de luminosidade. No entanto, propõe-se uma nova metodologia para estimativa do amortecimento de ondas por campos de vegetação que separa o problema, altamente complexo, em duas etapas. Na primeira, investiga-se o comportamento de uma única haste isolada, determinando coeficientes hidrodinâmicos a partir do movimento observado e das previsões teóricas para as velocidades e acelerações das ondas utilizando teoria não linear de alta ordem. Na segunda etapa, realizam-se os ensaios com o conjunto de hastes, utilizando-se as equações do movimento promediadas no tempo e integradas na vertical. Os coeficientes obtidos na primeira etapa são então incorporados nesta fase, permitindo assim quantificar a influência do conjunto em relação ao que ocorre com uma única haste. Esta metodologia representa um primeiro passo no sentido de investigar o complexo movimento de um conjunto de hastes e suas interações com o escoamento oscilatório, quantificando, de maneira o mais precisa possível, as forças incidentes sobre uma haste e, a seguir, incluindo este fator no amortecimento do conjunto.

Ao contrário de outros trabalhos encontrados na literatura, que utilizaram um "coeficiente de arraste" para o conjunto ou que procuraram modelar exclusivamente a força exercida em um elemento individual, a investigação experimental proposta no presente trabalho, que será apresentada na Parte II, procura quantificar a relação entre o efeito do conjunto e a força sobre uma haste isolada. A metodologia proposta permite ser generalizada para outros tipos de vegetação e investigar, de maneira racional, as relações entre os efeitos de conjunto e de uma única haste. Finalmente, por utilizar a teoria não linear da Função de Corrente, são levadas em consideração as assimetrias típicas dessas ondas, permitindo a aplicação da metodologia a diversas situações de profundidade relativa, desde águas intermediárias até águas rasas.

\section{CONCLUSÕES}

Apresentou-se um modelo de amortecimento de ondas de gravidade propagando-se através de um conjunto de elementos artificiais flexíveis. Conceitualmente, o fenômeno foi dividido em dois processos distintos. Inicialmente, a resistência individu- 
al de uma haste foi caracterizada através de um modelo mecânico que, através da equação de Morison, utilizando a velocidade relativa fluido-haste, calculou as forças exercidas pelas ondas sobre uma haste. Uma vez estimada a contribuição de força sobre um elemento, utilizaram-se as equações do movimento integradas na vertical e promediadas no período da onda para avaliar o amortecimento gerado pelo conjunto.

A segunda parte deste trabalho apresentará os procedimentos experimentais adotados para a calibração das forças através da determinação de um co-eficiente de arraste $\mathrm{CD}$, bem como do decaimento da onda através de um campo de elementos flexíveis.

A segunda parte deste trabalho apresentará os procedimentos experimentais adotados para a calibração das forças através da determinação de um co-eficiente de arraste $\mathrm{C}_{\mathrm{D}}$, bem como do decaimento da onda através de um campo de elementos flexíveis.

\section{AGRADECIMENTOS}

Este trabalho foi desenvolvido com recursos do Projeto CAPES/PROCAD 0144/01-0 - “Amigos de Boussinesq - Rede Cooperativa de Pesquisa sobre Hidrodinâmica Costeira de Águas Rasas", envolvendo a COPPE/UFRJ, a FEIS/UNESP e o I$\mathrm{PH} /$ UFRGS. Os autores agradecem especialmente ao INPH - Instituto de Pesquisas Hidroviárias, onde os ensaios experimentais foram realizados, e a Moacyr de Paula, do NCE/UFRJ, pelo desenvolvimento do programa de processamento de imagens.

\section{REFERÊNCIAS}

BREBBIA, C.A., WALKER, S., 1979, Dynamic analysis of offshore structures. 1 ed. London, NewnesButterworths.

BRUNO, J. F., KENNEDY, C. W., 2000, "Patch-size dependent habitat modification and facilitation on New England cobble beaches by Spartina alterniflora". Oecologia, v. 122, pp. 98-108.

CAMFIELD, F. E., 1983, "Wind-wave growth with high friction". Journal of Waterways, Port., Coastal and Ocean Engineering, v. 109, n. 1, pp. 115-117.

CARBONARI, C.A., MARTINS, D. TERRA, M.A., 2003, "Controle de Brachiaria subquadripara e Brachiaria mutica através de diferentes herbicidas aplicados em pósemergência". Planta Da-ninha, v.21, Edição Especial, pp.79-84.

CHAKRABARTI, S. K., 1987, Hydrodynamics of offshore structures. 1 ed. New York, Springer-Verlag.

COOPS, H. GEILEN, N. VERHEIJ, H. J., BOETERS, R., VELDE, G., 1996, "Interactions between waves, bank erosion and emergent vegetation: an experimental study in a wave tank". Aquatic Botany, v. 53, pp. 187198.

DALRYMPLE, R. A., KIRBY, J. T., HWANG, P.A., 1984, "Wave refraction due to areas of energy dissipation". Journal of Waterways, Port., Coastal and Ocean Engineering, v. 110, n. 1, pp. 67-79.

DEAN, R. G., 1968, Relative validities of water wave theories. In: Technical Progress Report No. 16, University of Florida, Gainesville.

DEAN, R. G., DALRYMPLE, R. A., 1984, Water wave mechanics for engineers and scientists. 1 ed. Englewood Cliffs, New Jersey, Prentice-Hall Inc.

DENNY, M. W., GAYLORD, B., COWEN, B., 1997, "Flow and flexibility II. the roles of size and shape in determining wave forces on the bull kelp nereocystis luetkeana". The Journal of Experimental Biology, v. 200, pp. 3165-3183.

DENNY, M., 1999, "Are there mechanical limits to size in wave-swept organisms ?". The Journal of Experimental Biology, v. 202, pp. 3463-3467.

DENNY, M., GAYLORD, B., 2002, "The mechanics of waveswept algae". The Journal of Experimental Biology, v. 205, pp. 1355-1362.

ELWANY, M. H. S., O'REILLY, W. C., GUZA, R. T., FLICK, R. E.,1995, "Effects of southern California kelp beds on waves". Journal of Waterways, Port, Coastal and Ocean Engineering, v. 121, pp. 143-150.

GAYLORD, B., DENNY, M. W., 1997, "Flow and flexibility I. Effects of size, shape and stiffness in determining wave forces on the stipitate kelps eisenia arborea and pterygophora californica". The Journal of Experimental Biology, v. 200, pp. 3141-3164.

GAYLORD, B., DENNY, M. W., KOEHL, M. A. R., 2003, "Modulation of wave forces on kelp canopies by alongshore currents". Limnology and Oceanography, v. 48 , n. 2, pp. 860-871.

IRGANG, B. E. e GASTAL Jr., C. V. de S., 1996, Macrófitas aquáticas da planície costeira do Rio Grande do Sul. 1 ed. Porto Alegre, Edição dos autores.

JOLY, A. B., OLIVEIRA FILHO, E. C., 1967, Two Brazilian Laminariales. In: Publicação especial, Instituto de Pesquisas do Mar, pp. 1-13.

KOBAYASHI, N., RAICHLEN, A. W., ASANO, T., 1993, "Wave attenuation by vegetation". Journal of Waterways, Port, Coastal and Ocean Eng. Div., v.119, n.1, pp. 30-48. 
LIMA, S.F., 2005. Amortecimento de ondas gravitacionais por campos de vegetação simulados por elementos de grande flexibilidade. Tese de Doutorado. Programa de Engenharia Oceânica, COPPE/UFRJ.

LIMA, S. F., NASCIMENTO, M. F., UAISSONE, A. J. R., NEVES, C. F., 2003, "Estimativa das maiores ondas geradas pelo vento no reservatório de llha Solteira". In: Anais do XV Simpósio Brasileiro de Recursos Hídricos, CD-ROM, Curitiba/PR.

LIMA, S. F., VASCO,J.R., NEVES, C.F., MACIEL, G.F., ROSAURO, N.M.L., 2005, "Simulation of fle-xible vegetation motion induced by water waves". In: Proc. Waves 2005, ASCE, CD-ROM, Madrid.

LONGUET-HIGGINS, M.S., 1976, "The mean forces exerted by waves on floating or submerged bodies, with applications to sand bars and wave power machines". Proc. Royal Society A, Vol. 106.

MADSEN, J. D., CHAMBERS, P. A., JAMES, W. F., KOCH, E. W., WESTLAKE, D. F., 2001, "The interaction between water movement, sediment dynamics and submersed macrophytes". Hidrobiologia, v. 444, pp. 7184.

MENDEZ, F. J., LOSADA, I. J., LOSADA, M.A., 1999, "Hydrodynamics induced by wind waves in a vegetation field". Journal of Geophysical Research, v. 104, n. C8, pp. 18383-18396.

MENDEZ, F. J., LOSADA, I. J., 2004, "An empirical model to estimate the propagation of random breaking and nonbreaking waves over vegetation fields". Coastal Engineering, v. 51, pp. 103-118.

MORK, M., 1996, Wave attenuation due to bottom vegetation. In: Waves and nonlinear processes in hydrodynamics, Kluwer Academic Publishers, pp. 371-382.

NEVES, C. F., 1987, A particular rotational wave theory. Ph.D. dissertation, University of Florida, Gainesville, Florida, USA.

OLIVEIRA, E. C., CORBISIER, T.N., ESTON, V.R., AMBROSIO JR. O., 1997, "Phenology of a seagrass (Halodule wrightii) bed on the southeast coast of Brazil". Aquatic Botany, v. 56, pp. 25-33.

PHILLIPS, O. M., 1977, The dynamics of the upper ocean. 2 ed. Cambridge, Cambridge Univ. Press.

PRICE, W. A., TOMLINSON, K. W., HUNT, J. N., 1968, "The effect of artificial seaweed in promoting the build-up of beaches". In: Proceedings of the 110 Coastal Engineering Conference, v. 1, pp. 570-578.

SEYMOUR, R. J., 1996, Discussion of "Effects of southern California kelp beds on waves", ELWANY, H. S., O'REILLY, W. C., GUZA, R. T., FLICK, R. E., Journal of Waterway, Port, Coastal and Ocean Eng. Div. v.122, n.4, pp. 207-208.

VASCO, J.R.G., 2005, Modelo conceitual de dissipação da energia da onda que se propaga por fundos vegetados. Dissertação de Mestrado. Programa de Pós-
Graduação em Engenharia Civil, Faculdade de Engenharia de llha Solteira, UNESP. 93p.

\section{Decay of Gravitational Waves by Fields of Flexible Vegetation. Part I - Theoretical Formulation of the Model}

\section{ABSTRACT}

This work presents a method for determining wave damping by vegetation fields. Other models found in the literature are restricted to plants which behave as rigid, fixed structures, therefore restricting their application to highly flexible plants such as Brachiaria subquadripora, commonly found along the margins of artificial lakes in Brazil. Conceptually, the physical phenomenon was divided in two processes. First, the resistance of a single stem is computed based on a mechanical model that uses the relative velocity between fluid and stem and an empirically adjusted drag coefficient, in order to compute the depth integrated, time averaged force on the stem. This expression is then introduced in the depth integrated, time averaged momentum equations for the fluid as a resistance term, and thus allowing to compute the wave height decay. The ensemble reaction against the waves is computed as a function of the average force on a single stem, of the spatial density of the plant individuals, and of an ensemble coefficient. The second part of this work will present the experimental procedures for calibrating the model and results. Key-words: gravitational waves; drag coefficient. 